\title{
Measures and visualization methods of map projection distortions with the use of "python matplotlib library" as an example
}

\begin{abstract}
The aim of the author of this article is to show the users of Geographical Information Systems how to present the distortions in a simple way. The intention of the author is also to popularize the knowledge in the scope of map projections and to inform the users of the maps that, despite all the advanced modern tools, an elimination of the problem, connected with the map projections and cartographical distortions occurring in them, has failed so far.

The author presents a brief overview of the measures in the scope of distortions and methods of their presentation. It is also shown how the users can generate the maps, presenting a distortion by themselves. It is much easier to perform this type of visualization with the help of "matplotlib library", basically everyone can compile such a map.
\end{abstract}

Keywords: map distortions measures, methods of presentations, Tissot's ellipses, distortion isograms, programming language "python", "matplotlib library"

\section{Introduction}

A huge variety of cartographical projections can cause a lot of troubles for the users and creators of geographical information systems. Each map projection is characterized by different properties. The shape of graticules, distribution of distortions may vary significantly from one another, and the dimension of distortions in a significant way may affect the analytical results and calculations. Any GIS user may face the problem of choosing a projection and solving the problem how to deal with the distortions occurring in this projection. A possible bad choice of a projection can cause many problems (fig. 1).

So, use the projections with the full awareness of distortions contained in them. The distortions are the inherent characteristics of the maps. They arise because of the impossibility of developing the earth's surface on the plane. Each map projection has its distortions and every cartographer, map maker or creator of spatial databases and Geographical Information Systems should has an ability to study them.
According to that, what F. Biernacki wrote (1949): "An essence of the scientific treatment of projections is an examination of damages in their
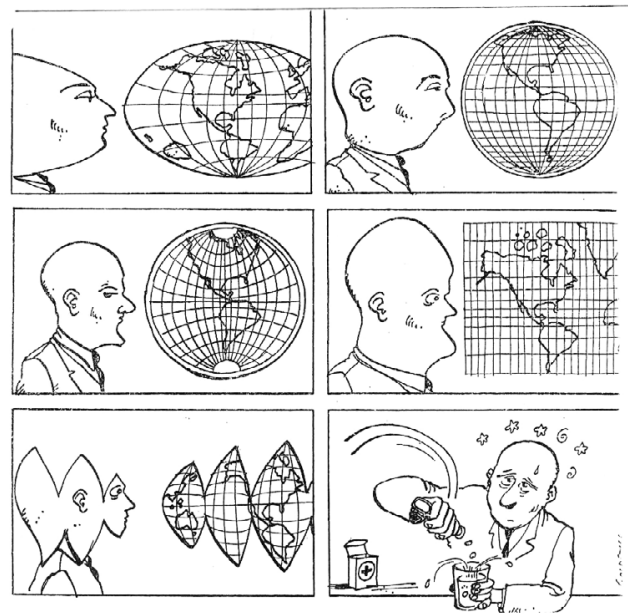

Fig. 1. The distortions can be a source of many problems (source: Jacques Goldstyn, 1983, sandbox.idre.ucla.edu/sandbox/intro-to-maps-historyand-beyond) 
metrics or metric distortions caused by the projection of one surface to another. In order to have an assessment of projection in terms of its metric properties, so valuable in both, theory and practice, you should study what, where, how and how much is distorted as the result of the process of projection". Using the map projections, it is necessary to know their properties. Today, in the era of maps commonly compiled by the unprofessional cartographers-amateurs, we often forget about the problem of distortions. The simplicity and general availability of tools, which are necessary for map compiling and database developing, mean that virtually everyone can compile a map, but perhaps not everyone knows that it has the distortions. Therefore, the analysis and calculations implemented on a map without the knowledge of the principles of cartometry may represent the considerable errors and a source of incorrect conclusions. The results of research on this type of issues are presented, among others, in the work (N. Chrisman and J.F. Girres 2015).

The cartographical projections of one surface to another, and in particular to the plane, always have the distortions. Depending on a size, which is measured on a map, we distinguish the distortions of length, angles and area. We can determine such the distortions in any cartographical projection with the defined projecting functions. The theory of distortion was developed and described in 1881 in Paris by A. Tissot in his work Memoire sur la representation des surfaces et les projections des cartes geographices (https://archive.org/details/mmoiresurlarepr00tissgoog). There are a number of measures used to study the distortions, which we can use. Some measures allow us to specify the properties of projections, the other ones to make corrections to the calculated sizes. The distortions can be presented on a map with the different methods of presentation. The most important are the ellipses and isolines of distortion, but there are also a number of other interesting methods, which will be presented in the article later. The aim of this publication is to highlight the issues connected with the research of metric properties of the cartographical projections by using the modern computer tools. The measures of distortions and methods of their presentation, as well as the computer programs, that can be used to study the distribution of distortions, will be described.
Some samples of scripts made by authors in python, operating in the environment called QGIS, will be also presented. The "matplotlib library", which allows you to perform in a very quick and easy way the visualization of distortion distributions, is used to develop the scripts.

\section{The classification of distortion measures}

A number of measures serves to study the distortions. We can divide them into local-directional, local and global. The local-directional measures are those, which depend on the position of a point and the direction at this point, the local measures depend only on the position of a point, while the global measures characterize the distortion in the entire projected area.

The local-directional measures include the elementary scale of length distortion, which is defined as the ratio of the corresponding elementary arcs on the surface of an original and an image. The scale of length distortion is a function of three variables, i.e., the coordinates defining the position of a point and the direction at a given point.

In the case of projection of the sphere into the plane, the pattern of the scale of length can be written as:

$$
\vec{\mu}=\vec{\mu}_{\varphi} \cos A+\vec{\mu}_{\lambda} \sin A
$$

Vector figure is a very convenient to use, because it gives us the information about the value and the direction of the determining scale.

In the above formula, $A$ is a geographic azimuth, and $\vec{\mu}_{\varphi}, \vec{\mu}_{\lambda}$ are the so called parametrical scales, i.e., the scales of the distortions of length designated in the direction of the parametrical lines, i.e., latitude and longitude. These scales are calculated from the formulas:

$$
\vec{\mu}_{\varphi}=\frac{\vec{r}_{\varphi}^{\prime}}{R}, \vec{\mu}_{\lambda}=\frac{\vec{r}_{\lambda}^{\prime}}{R \cos \varphi},
$$

where $\vec{r}_{\varphi}^{\prime}, \vec{r}_{\lambda}^{\prime}$ are the partial derivatives of the projecting functions in terms of the geographical coordinates.

As the scale of distortions depends on the direction at a given point, we can look for such a direction, in which the scale reaches the extreme. In the scope of cartographical projec- 
tions, we have four such the directions that differ by $90^{\circ}$. We calculate them from the formula:

$$
\tan \left(2 A_{e}\right)=\frac{2 Q}{P-R}
$$

where:

$$
Q=\frac{F^{\prime}}{\sqrt{E G}}, \quad P=\mu_{\varphi}=\frac{E^{\prime}}{E}, \quad R=\mu_{\lambda}=\frac{G^{\prime}}{G},
$$

wherein $E, F, G$ are the coefficients of the first quadratic form of an original surface and $E^{\prime}, F^{\prime}$, $G^{\prime}$ are the coefficients of the first quadratic form of an imaginal surface.

The scale of the distortion of length in the conformal projections do not depend on the direction at a given point, so:

$$
\mu=\mu_{\varphi}=\mu_{\lambda}=m=n,
$$

wherein $m$ and $n$ are the extreme scales.

Within the equal-area projections, the extreme scales are the inverses of each other, so

$$
m=\frac{1}{n}
$$

The scale of the distortion of length is the ratio of the corresponding elementary arcs at the surface of an image and at the surface of an original, so if the scale is equal to one, this means, that the arcs are equal and there is no distortion in length. If a scale is less than a unity, it means the contraction of an original surface, eg. the scale of 0.5 means the reduction of length of section twice, but if a scale is greater than a unit, it means the tension of the surface of an original, eg. when the scale is equal to 2 , it means the elongation of infinitesimal section two times.

Sometimes as a measure of distortion is used the natural logarithm of the scale of length. This measure has the property of symmetry. Eg. the scale of 0,5 after taking the logarithm is $-\ln 2$, while the distortion of the scale which is equal to 2 is $\ln 2$. For scale equal to 1 distortion is 0 .

Another local-directional measure is a local distortion construed as the deviation of the scale of length from a unity. If the scale is equal to 1 , the distortion is equal to 0 , when a scale is less than a unity, the distortion is negative, and when a scale is greater than a unity, the distortions are positive. The distortions defined in such a way are usually expressed in the relative form, which means a change in length of a unit section, for example, $\mathrm{m} / \mathrm{km}, \mathrm{cm} / \mathrm{km}$, $\mathrm{cm} / 100 \mathrm{~m}$.

Distortions at any point and in any direction can therefore be considered in various forms:

$$
z_{1}=\mu-1, \quad z_{2}=\ln \mu,
$$

i.e., these well-known, and

$$
z_{3}=1-\frac{1}{\mu}, \quad z_{4}=\frac{1}{2}\left(\mu^{2}-1\right),
$$

which are less popular.

The local measures include also the measures that characterize the length of distortion at a given point, regardless of direction. Thus, they may be eg., the extremal scales, or for example, the average value of the extremal scales or distortions at a given point,

$$
\mathcal{E}=\frac{1}{2} \sqrt{(m-1)^{2}+(n-1)^{2}}
$$

An interesting modification of this measure was presented by Kimerling, Overton, White (1995) in the form of

$$
\mathcal{E}=\sqrt{m^{2}+n^{2}}-\sqrt{2}
$$

In their article, they dealt with the application of statistics to study the distortions.

To assess the value of distortions of the areas, the scale of distortions of the areas is most widely used

$$
p=m n=\frac{H^{\prime}}{H},
$$

where $H$ and $H^{\prime}$ are the determinants of the first quadratic form of the surfaces of an original and image.

As in the case of distortions of length, also the distortions of the areas can be determined by the deflection of a scale of distortions in the areas from a unity. The result, as in the case of distortions of length, can be written in the relative form, for example: $\frac{\mathrm{m}^{2}}{\mathrm{~km}^{2}}$ or expressed in the percent or parts per thousand, depending on the obtained value of a distortion.

A measure of the distortion of angle is usually the doublled value of the extremal distortion of the directional angle: 


$$
z_{k}=2 \arctan \left(\frac{m-n}{2 \sqrt{m n}}\right)
$$

The distortion of any angle in a given point does not exceed the value determined by the above formula.

The measures, which characterise the distortions in the entire projected area, are included to the global measures. Most often the value of distortion is calculated by the formula:

$$
E^{2}=\frac{1}{F} \int_{F} \varepsilon^{2} d F
$$

where $\varepsilon$ means the local measure and $F-$ a field of an area.

The approximate value can be calculated as follows: it is necessary to divide the area into several small sub-areas, for which in the central point an $\varepsilon^{2}$ should be calculated using the formulas for local measures, and then one should add up the calculated values:

$$
E=\sqrt{\frac{1}{k} \sum_{i=1}^{k} \varepsilon_{i}^{2}}
$$

Among the integral measures we can distinguish (J. Balcerzak, J. Panasiuk, U. Pokrowska 1995, P. Pędzich 2008):

- Airy's measures

$$
E_{A}^{2}=\frac{1}{F} \int_{F}\left[(m-1)^{2}+(n-1)^{2}\right] d F ;
$$

- Fiorini's measures

$$
E_{F}{ }^{2}=\frac{1}{F} \int_{F}\left[\left(\frac{m}{n}-1\right)^{2}+(m n-1)^{2}\right] d F \text {; }
$$

- Jordan's measures

$$
E_{J}^{2}=\frac{1}{F} \int_{F}\left[(\ln m)^{2}+(\ln n)^{2}\right] d F,
$$

wherein $m$ and $n$ are the extreme length scales or length scales in the main directions of cartographic projection.

These measures may also serve as the criteria for determining the projections of given areas.
It is requested then that a distortion calculated according given measure is the smallest.

According to L. Bugejewski and J. Snyder (1995) there are such criteria:

Airy

$$
\begin{gathered}
E_{A}{ }^{2}=\frac{1}{F} \int_{F}\left[(m-1)^{2}+(n-1)^{2}\right] d F ; \\
E_{F}{ }^{2}=\frac{1}{F} \int_{F}\left[\left(\frac{m}{n}-1\right)^{2}+(m n-1)^{2}\right] d F ;
\end{gathered}
$$

Airy-Kawrajski

$$
E_{J}{ }^{2}=\frac{1}{F} \int_{F}\left[(\ln m)^{2}+(\ln n)^{2}\right] d F ;
$$

Jordan

$$
E_{J}^{2}=\frac{1}{2 \pi} \int_{0}^{2 \pi}(\mu-1)^{2} d \alpha
$$

Jordan-Kawrajski

$$
E_{J-K}^{2}=\frac{1}{2 \pi} \int_{0}^{2 \pi} \ln ^{2} \mu d \alpha ;
$$

Klingach

$$
E_{F}{ }^{2}=\frac{1}{F} \int_{F} \frac{P_{\omega}\left(\frac{m}{n}-1\right)^{2}+P_{p}(m n-1)^{2}}{P_{\omega}-P_{p}} d F,
$$

where with the help of weights $P_{\omega} \mathrm{i} P_{p}$ we can establish the balance between the distortion of angle and surface areas.

Konusow:

$$
\alpha=\arctan \frac{\frac{m}{n}-1}{m n-1},
$$

with the help of which we can estimate or set up a distortion in the conformal projection: $\alpha=\frac{\pi}{2}$ for the equal-areas projections and $0<\alpha<\frac{\pi}{2}$ for any projections.

\section{Methods of the distortion presentation}

There are many presentation methods of distortions. The most famous and widely used 
methods are isolines of distortion and Tissot's indicatrix. The presentation methods of distortions can be divided into illustrative ones, i.e., those, which are used mainly for teaching purposes and therefore only highlight how significant the distortions are in this or that projection and analytical methods for scientific purposes, the aim of which is to present on a map both, the size of the distortions in a given place, as well as their distribution over the projected area. Many interesting presentation methods of distortions described K.A. Mulcahy and K.C. Clarke (2001). The authors didvided them into 10 groups, among them is a presentation of distortion using the familiar shapes: squares, checker, cartographical grids, by comparing a size of the projected areas, isolines, using color, Tissot's indicatrix, perspective visualizations of the surface of distortion and interactive presentation of distortion.

Interesting work on the analysis and presentation of distortions was published by P. Laskowski (1997). Creator of the notion of distortion spectrum proposed his own method and discribe a number of different methods of distortion presentation. P. Laskowski's method allows not only to compare any projection with another one in the scope of size of total distortions, but also to compare the size of distortions of different types: arcs, length, surface areas. It means that the root mean square measures of distortions are calculated in the reference projection, and then, the inverses of these measures are used as the calibration coefficients for the measures in the comparative projections, that means, that a user multiplies a value of distortion in a given projection by the coefficient calculated in the reference projection. In this way, we get rid, among others, of the units in the indicated distortions. This gives the result in the so-called standard units of distortion - SUD (Standard Unit of Distortion). This gives the users an opportunity to compare the size of distortions of various types, e.g., distortion of angles and distortion of surface areas, but primarily it allows to compare different projections with each other. P. Laskowski (1997) proposed to present the calculated values of distortions on the graph or on the diagram. The total value of these distortions in two different projections allows to compare these projections with respect to the size of distortions.
W. Tobler was also involved into the studying of similarities in the scope of projections. A method based on the calculation of "the distance" projections was developed (W. Tobler 1986). The results of his calculations for the projection of Sanson and three other projections were presented by him with the help of a map, on which he drew the distances between the nodes of graticules corresponding to each

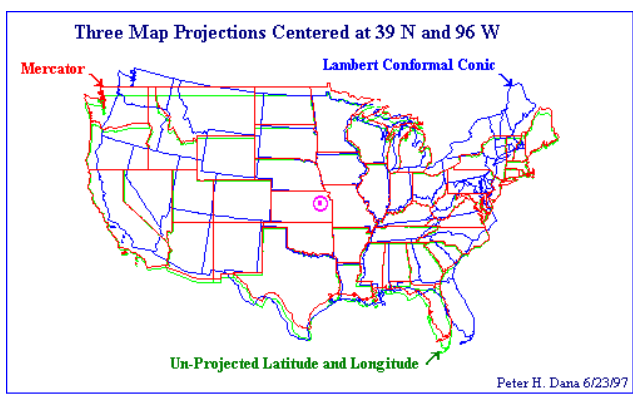

Fig. 2. A map showing the differences in the shape of an area of the US in various cartographical projections (source: http://www.colorado.edu/geography/gcraft/notes/mapproj/mapproj_f.html)

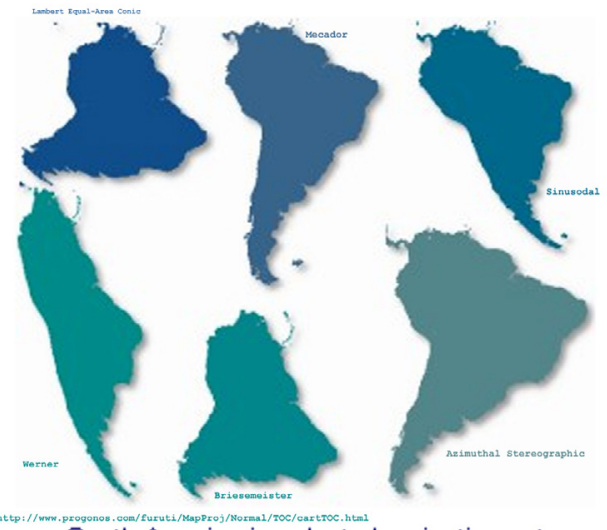

South America in selected projections at identical scale. Which projection is best? Which is right? The short answer is none, at least not all the time. Even if a single projection is used, just switching the aspect can also radically reshape the continents.

Fig. 3. An illustration of South America in a variety of cartographical projections

(source: http://www.progonos.com/furuti/MapProj/ Normal/TOC/cartTOC.html) 
other in different map projections by means of straight lines.

Distortion can be visualized using a variety of objects presented on the maps, they can be physiognomies, faces, contours of objects. For example, in figure 2 we can see an overlay of contours of the United States. Illustrated in three different projections by means of different colors, they show the distortions of boundaries very well.

In figure 3, in turn, it is presented an outline of South America in various cartographical point (fig. 4), but also the global distribution of distortions in a given area (fig. 5).

One more way of presenting the distortions are the isolines of distortion. They may have a form of lines of the same color or of different colors, depending on the distortion (fig. 6). The isolines of distortion can present the various types of distortion, both, the length and the areas, and the angles, as well. Additionally, to increase the drawing, the isolines of distortion can be completed with some color, filling the space between the isolines of distortion (fig. 7).
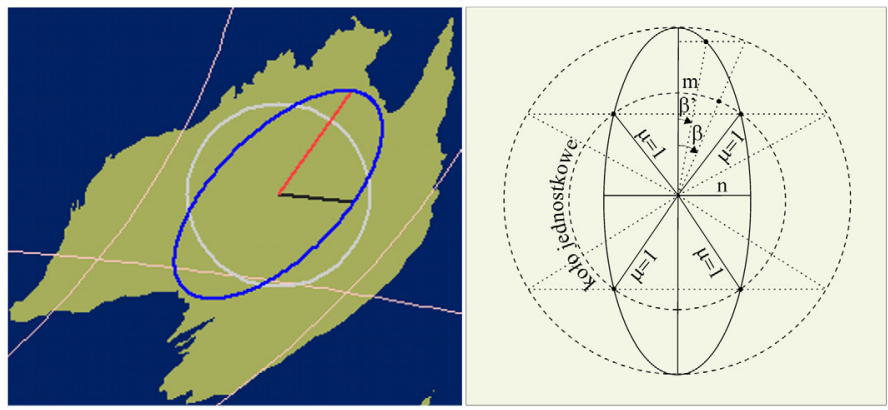

Fig. 4. The presentation of the local distortions using the Tissot's indicatrix in the neighborhood of a point (source: http://www.uni-stuttgart.de/gi/geoengine/mappro/ and P. Pędzich 2014)

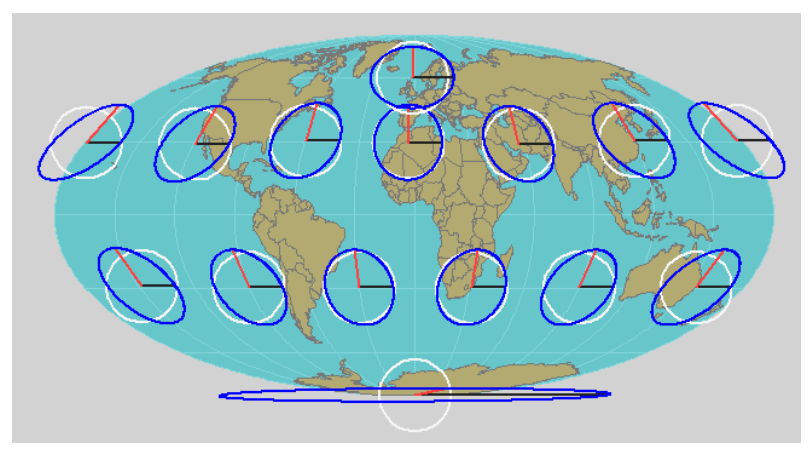

Fig. 5. The presentation of the distribution of distortions using the Tissot's ellipses (source: http://www.uni-stuttgart.de/gi/geoengine/mappro/)

projections. This figure gives us a pretty good indication as to the size of distortions on the maps in different cartographical projections.

Another way of presenting the distortions are Tissot's indicatrices. This well-known method, described in many textbooks, allows us to present the local distortions around a given
The choice of colours is very important here. With the reduction of distortion increases the brightness in the case of using one colour.

In the case of using the scale of colours, different values of distortions are assigned to different colors, for example, green is assigned to the low distortion, red to the high distortion 

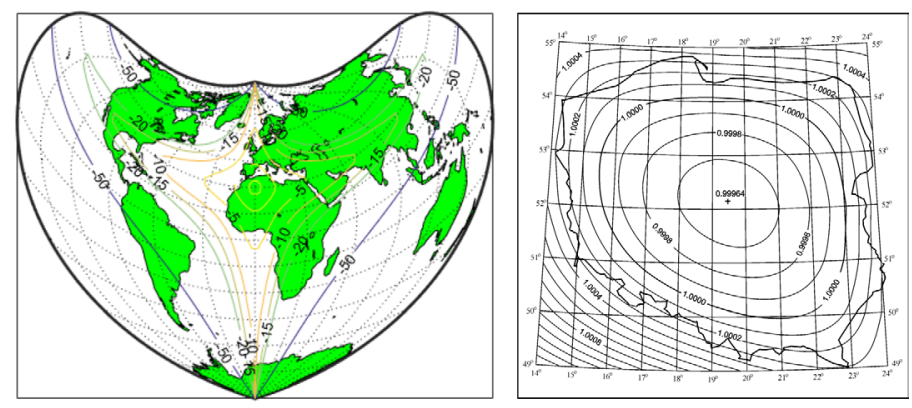

Fig. 6. The presentation of the distribution of distortions with the help of isolines of distortion (source: http:// www.mathworks.com/help/map/visualizing-and-quantifying-projection-distortions.html and P. Pędzich 2005)
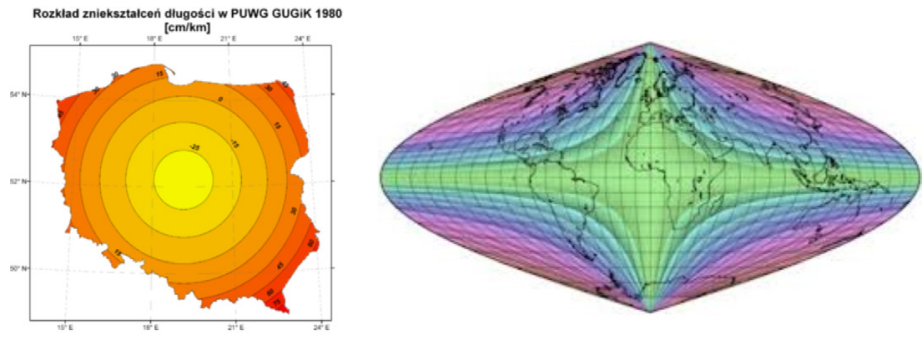

Fig. 7. The presentation of the distribution of distortions using the isolines and scale of colours (source: https://pl.wikipedia.org/wiki/Uk\%C5\%82ad_GUGIK_1980\#/media/File:Gugik80_znieksztalcenia.png and http://www.progonos.com/furuti/MapProj/Dither/CartProp/DefPatA/defPatA.html)

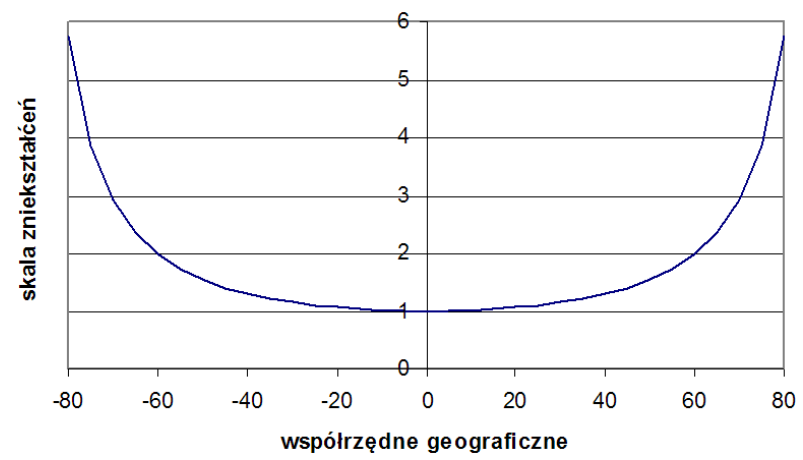

Fig. 8. A graph of the scale of distortions in the Mercator Projection

The graphs are often used for the presentation of distortions (fig. 8). If the distortions depend only on one variable, eg. the latitude, such a representation of the distribution of distortions is quite sufficient.
Since the distortions change with the position of a point on the map, we can also use the three-dimensional methods of presentation. Then, we put the rectangular coordinates on two axes of a system, and on the third axis - 


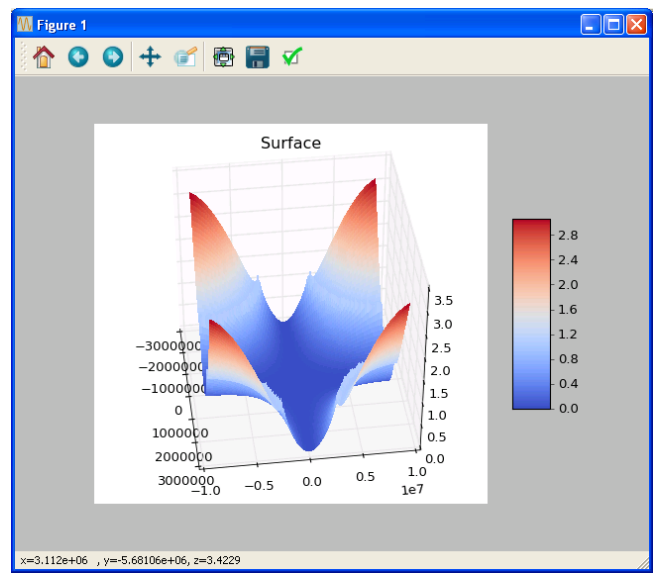

Fig. 9. The area of distortions in the Sanson projection

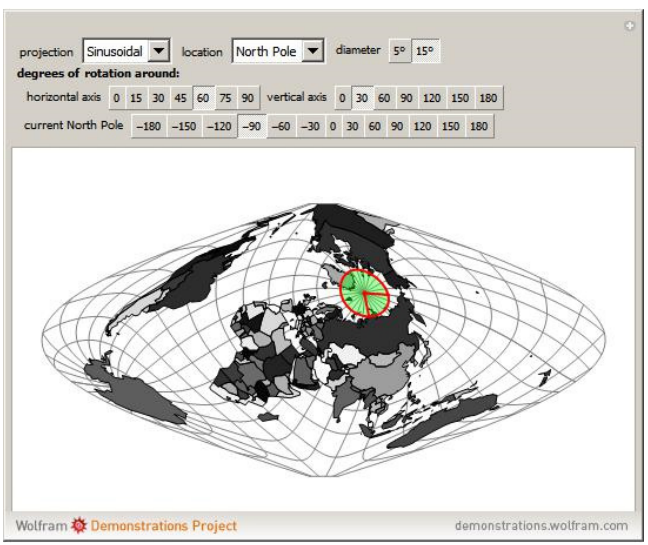

Fig. 10. The window of application of the Wolfram demonstration (source: http://demonstrations.wolfram.com/DistortionsInMapProjections/)
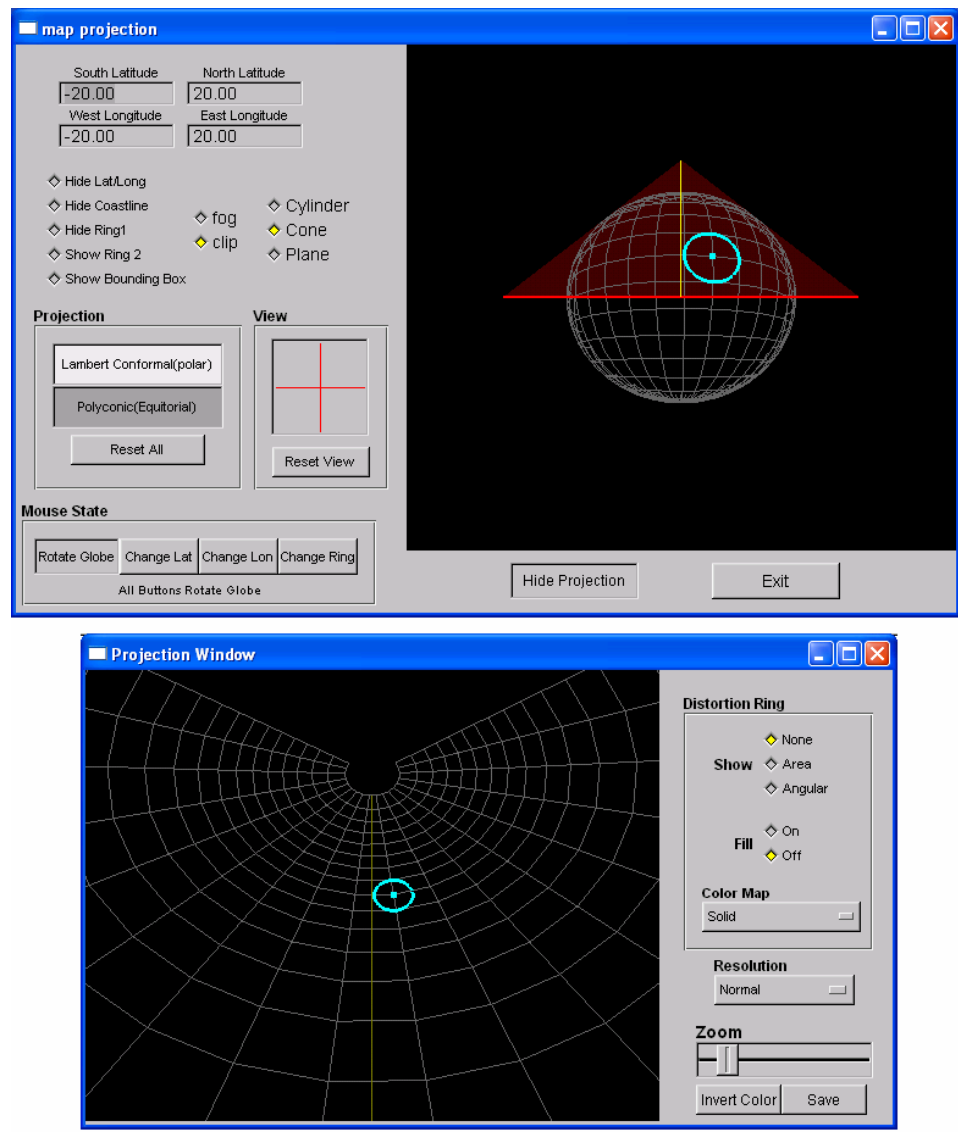

Fig. 11. A window of the mapwin application 
a value of distortion. An example might be a surface of distortions generated in the Sanson projection presented in figure 9.

With the development of various computer visualization techniques, there are also such studies, which allow for interactive presentation of distortion.

On http://demonstrations.wolfram.com/DistortionsInMapProjections/ one can find a program for interactive presentation of distortion in different cartographical projections. It is also shown how the distortions will change, if a map is transformed into the oblique projections while changing the parameters of these projections. This program allows you to select the several parameters, such as a type of projection, location of the ellipse of distortions, a size of the ellipse, the parameters of the oblique projections (fig. 10).

Another example of an application designed for the interactive presentation of distortions is an application called mapwin. This program displays the ellipse of distortions in a few selected projections. The parameters of projection, that you can configure, are as follows: type of projection (cylindrical, conical, azimuthal), central meridian. After clicking "show projection", the cartographical grid appears with the Tissot ellipse drawn (fig. 11). The application can be downloaded from the http://slvg.soe.ucsc.edu/ map.html.

On the http://projections.mgis.psu.edu/ you can use the interactive atlas of cartographic projections, written by Ryan Baxter of the University of Pennsylvania. The atlas can view a map of the world in a few selected projections. There are the outlines of the continents on the map, you can also display a graticule and the Tissot ellipses in the nodes of this graticle. Use the slider to change the meridian axis. In addition, you can select a desired location on the map (fig. 12).

We can also distinguish the puzzles in the WebMercator Projection from the other interesting developments related to the topic of presentation. Made on the basis of Google Maps, they allow us to see how huge distortion is introduced by this "innovative" solution from Google. In addition to the fun, which consists in laying the selected areas in the right place, we can also make a comparison of the selected areas and see to what extent the size of the area

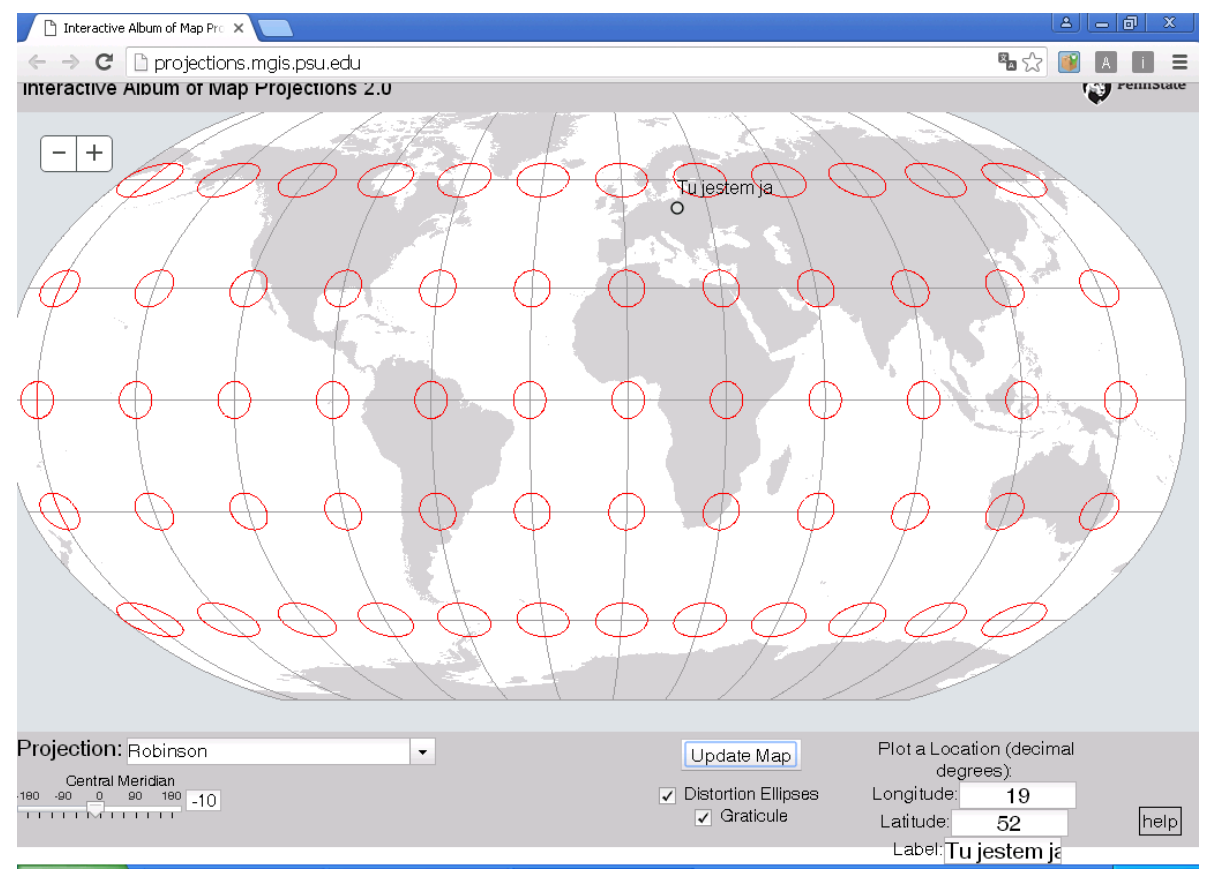

Fig. 12. The Interactive atlas of cartographical projections (source: http://projections.mgis.psu.edu/) 


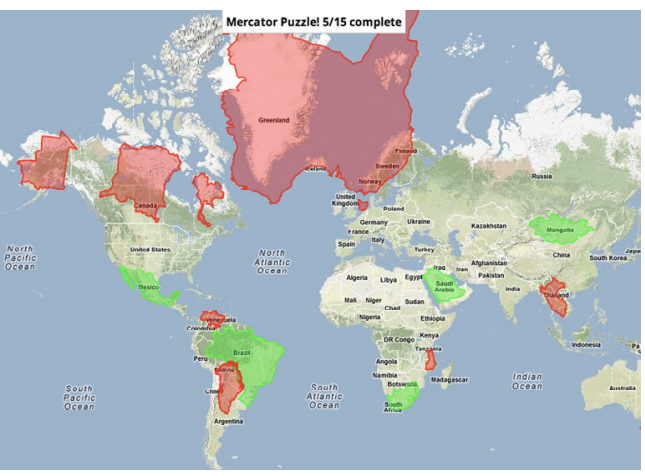

Fig. 13. The Puzzles of WebMercator (source: www.oshermaps.org/of-maps-and-history/ great-interactive-tool-to-teach-the-distortions-of-themercator-projection)

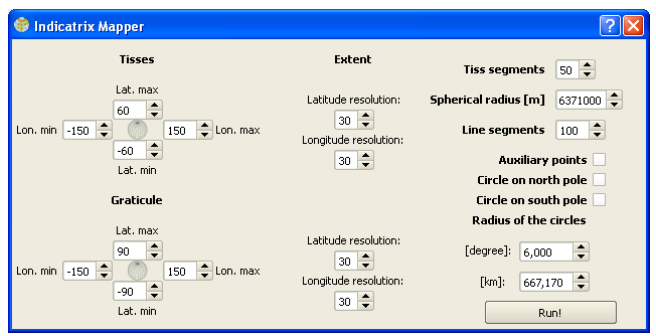

Fig. 14. A dialog window of the Indicatrix Mapper plug-in

changes together with the change of a position on the map (fig. 13).

A plug-in into the program called QGIS (fig. 14) developed by Ervin Wirth from the Budapest University of Technology and Economics is worth to mention among other useful tools for the visualization of distortion. It allows us to generate a graticule and the Tissot's ellipses in the nodes of this graticule. The plug creates two layers: a graticule is drawn on the first of them, the ellipses of Tissot - on the second. These layers can be combined with the others, which allows us to create the maps presenting the distribution of distortion. The disadvantage of this solution is that the ellipses of Tissot are not really generated by the program: some ovals, resulting from the projection of the spherical small circles on a plane, are generated. But roughly, this program shows quite correct distribution of distortions in the selected area of cartographical projection. This program has some problems with drawing a graticule.

\section{Presentation of distortions using matplotlib}

Matplotlib is a library in python that allows to perform the graphs of a very high-quality in a variety of formats and interactive environments across the different platforms. Matplotlib can be used in python, and ipython shell scripts (a'la MATLAB or Mathematica), Web application servers and sets of GUI tools. This library allows us to generate a variety of diagrams and graphs with the help of a few lines of code. The interface is similar to Matlab. For more advanced users there is a possibility of changing the line styles, font types, properties of the axes of coordinates, etc. through the many features known for the users of Matlab (http:// matplotlib.org/).

The capacity of the library can be used to visualize the distortions in cartographical projections. In conjunction with the QGIS, we get a tool that allows us to generate the ellipses of Tissot, isolines of distortion and three-dimensional presentations of distortions quickly and easily. On the website http://matplotlib.org/users/ screenshots.html\#mplot3d one can find a description of the functions and illustrations showing the different types of diagrams, achievable using the library matplotlib.

The use of the matplotlib library to present the distribution of distortions on the maps requires the basic knowledge of programming in python, as well as the knowledge of functions, which allows to use the basic capabilities of QGIS or other GIS program via the API. There are a number of tools to support the creation of plug-inns python, e.g. in QGIS we can take the advantage of the Plugin Buider that allows us to generate the basic code of new plug-in. Among the automatically generated files, there is one called nazwawtyczki_dialog.py, where you should enter the correct code to perform the appropriate calculations. The QTDesigner tool for creating the interface of application is attached to QGIS. If you press the button of the interface, a signal clicked (), pushed (), released () is sent, which gives the ability to perform the calculations by pressing the corresponding button on the interface. The source code to be executed by 


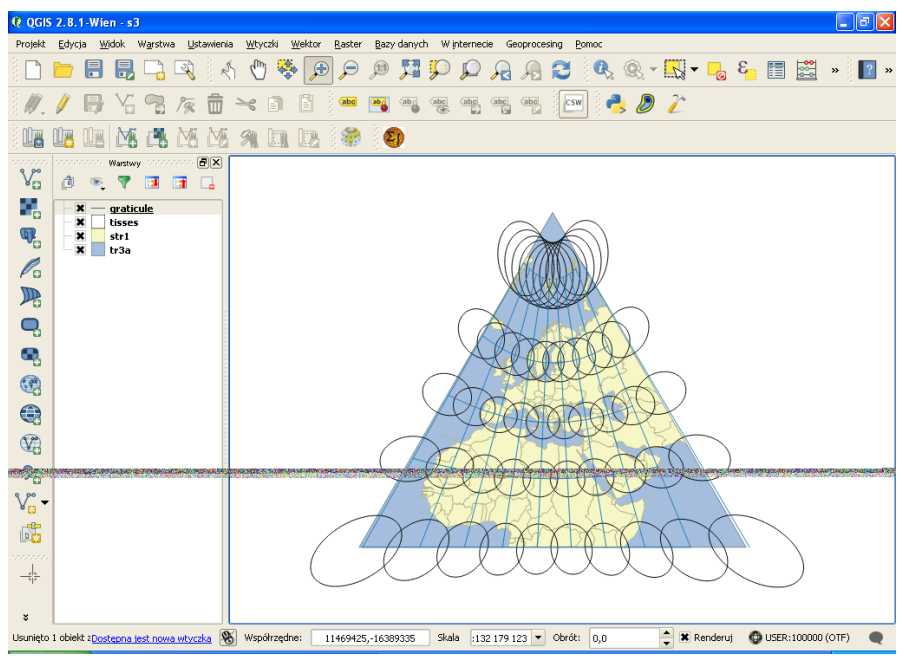

Fig. 15. A map developed in QGIS as a result of using the Indicatrix Mapper Plug

pressing the button is a part of a file nazwawtyczki_dialog.py after the method name called on_nazwaObiektu_sygnat (). An example script for generating the isolines of distortion for Airy measure at the currently set projection in QGIS for the active layer presents the way of creation of such a plug.

The main problem arising during the calculation is to determine the appropriate partial derivatives. The differential method for the determination of derivatives is used in the example. A sample source code, the enforcement of which will generate the isolines of distortion may take the form of:

def on_dlzol_released(self):

odwz $=$ qgis. utils.iface.activeLayer().crs()

$\mathrm{R}=6371000.0$

eps $=0.0001$

crsSrc $=$ QgsCoordinateReferenceSys tem(4326) \# WGS 84

crsDest $=$ QgsCoordinateReferenceSystem(odwz.toWkt())

xform = QgsCoordinateTransform(crsSrc, crsDest)

fip=self.latp.value()

fik=self.latk.value()

lamp=self.longp.value()

lamk=self.longk.value()

delta_f=self.dfi.value()

delta_I=self.dlam.value() delta $=100$

delta2 $=100$

$\mathrm{f}=\mathrm{np}$.linspace(fip,fik, delta)

lam=np.linspace(lamp,lamk,delta2)

$\mathrm{ptp}=x$ form.transform(QgsPoint(lamp,fip))

ptk $=$ xform.transform (QgsPoint $($ lamk,fik))

$x=$ np.linspace $($ ptp. $x()$, ptk.x(), delta2)

$y=n p$. linspace(ptp.y(), ptk.y(), delta)

$X, Y=n p$.meshgrid $(x, y)$

$Z=[[0$ for $i$ in range(delta) $]$ for $j$ in

range(delta2)]

for $\mathrm{i}$ in range(delta):

for $\mathrm{j}$ in range(delta2):

$f i 1=f[i]+e p s$

fi2=f[i]-eps

$\operatorname{lam} 1=\operatorname{lam}[j]+e p s$

lam2=lam[j]-eps

ptfi1 $=$ xform.transform(QgsPoint(lam[-

j],fi1))

ptfi2= xform.transform(QgsPoint(lam[-

j],fi2))

ptl1=xform.transform(QgsPoint(lam1,f[i]))

ptl2=xform.transform(QgsPoint(lam2,f[i]))

x_fi=(ptfi1.x()-ptfi2.x())/(2.0*eps*math.

$\mathrm{pi} / 180.0)$

y_fi=(ptfi1.y()-ptfi2.y())/(2.0*eps*math.

$\mathrm{pi} / 180.0)$

x_I=(ptl1.x()-pt|2.x())/(2.0*eps*math.

$\mathrm{pi} / 180.00)$

y_l=(ptl1.y()-pt|2.y())/(2.0*eps*math.

$\mathrm{pi} / 180.0)$ 


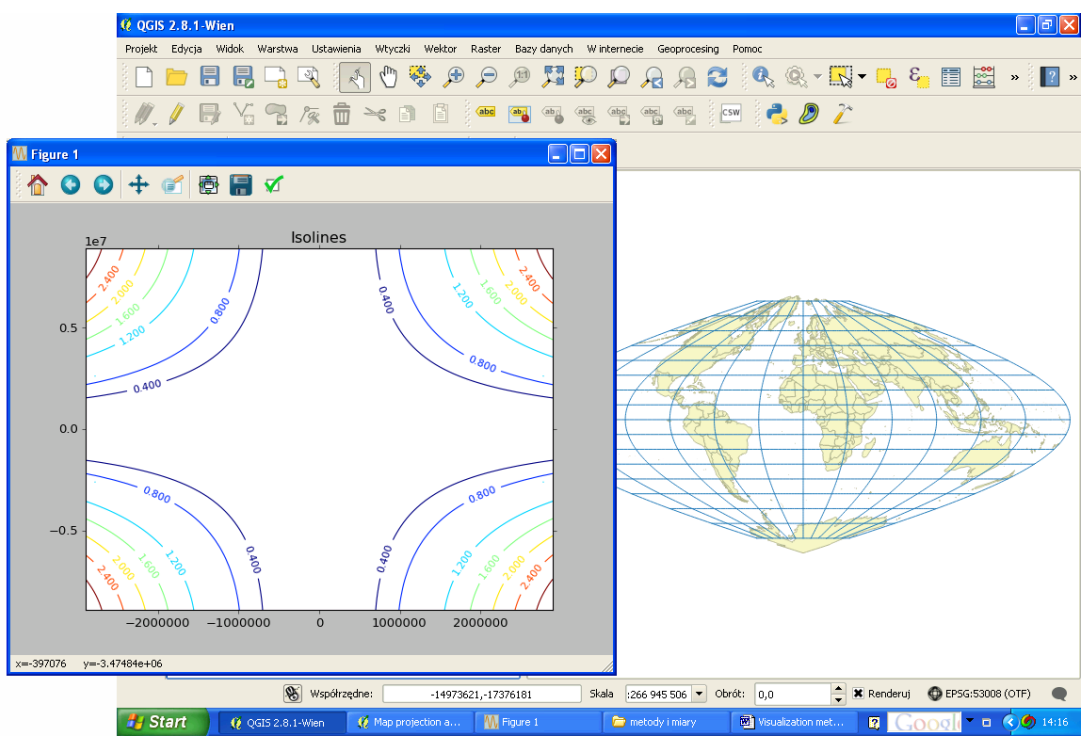

Fig. 16. The isolines generated by the developed plug-in

mifi=math.sqrt $\left(x_{-}\right.$fi ${ }^{*} x_{-} f i+y_{-} f^{*}{ }^{*}$

fi)/6371000.0

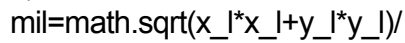

(6371000.0*math.cos(f[i] ${ }^{*}$ math.pi/180.0))

$\mathrm{fp}=\mathrm{x} \_\mathrm{fi}{ }^{*} \mathrm{x} \_\mathrm{I}+\mathrm{y} \_\mathrm{fi}^{*} \mathrm{y} \_\mathrm{I}$

$q q=f p /\left(6371000 \cdot 0^{*}\right.$ math $\cdot \cos \left(f[i]^{*}\right.$ math .

$\mathrm{pi} / 180.0)$ )

aee=math $\cdot \operatorname{atan}\left(q q /\left(\right.\right.$ mifi $^{*}$ mifi-mil $\left.\left.{ }^{*} m i l\right)\right)$

$\mathrm{m}=$ math.sqrt (math.fabs $\left(\mathrm{mifi}^{*}{ }^{*} \mathrm{mifi}^{*}\right.$ math. $\cos (\text { aee })^{*}$ math.cos(aee)

$+q^{*}$ math $\cdot \sin \left(2^{*} a e e\right)+$ mil $^{*}$ mil ${ }^{*}$ math .

$\sin (\text { aee })^{*}$ math.sin(aee)))

$\mathrm{n}=$ math.sqrt(math.fabs $\left(\right.$ mifi $^{*}$ mifi $^{*}$ math.

$\sin (\text { aee })^{*}$ math.sin(aee)-

$\mathrm{qq}^{*}$ math $\cdot \sin \left(2^{*}\right.$ aee $)+$ mil $^{*}$ mil ${ }^{*}$ math .

$\cos (\text { aee })^{*}$ math.cos $($ aee $\left.\left.)\right)\right)$

airy $=$ math.sqrt $\left((m-1)^{*}(m-1)+(n-1)^{*}(n-1)\right)$

$Z[i][j]=$ airy

matplotlib.rcParams['xtick.direction'] = 'out' matplotlib.rcParams['ytick.direction'] = 'out' fig=plt.figure()

$\mathrm{CS}=$ plt.contour $(\mathrm{X}, \mathrm{Y}, \mathrm{Z})$

plt.clabel(CS, inline $=1, \quad$ \# add labels

fontsize=10, \# label font size

)

plt.title('Isolines')

\# label locations

plt.show()
The name of the projection of the active layer is being read in the first line of this code. Then, the radius of the sphere and the increment of geographical coordinates for the calculation of derivatives are given. The next three lines of this code develop a transformation of coordinates between the projections. The next eight lines constitute a definition within the geographical coordinates of the area, for which we

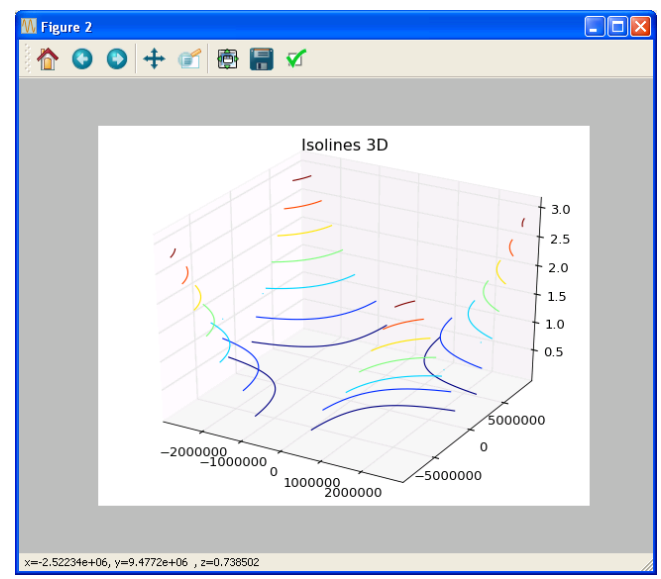

Fig. 17. The three-dimensional isolines generated by the developed plug-in 


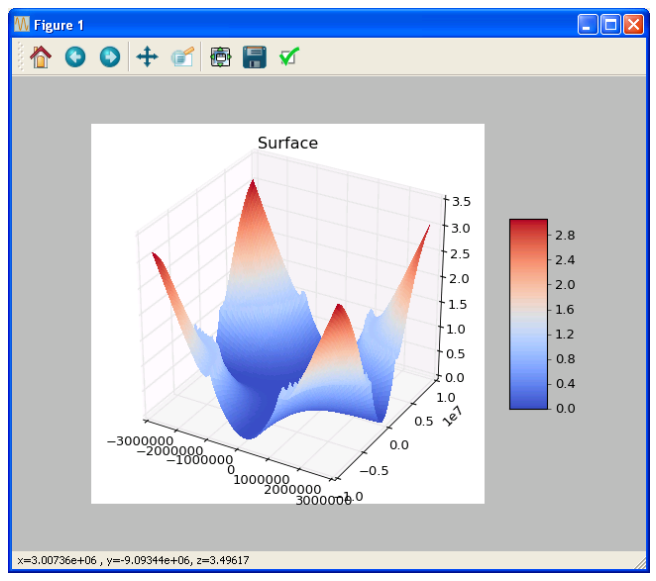

Fig.18. The surface of distortions with the scale of colours generated by the developed plug-in

perform the calculations. The next two lines are to generate the vectors with the values of geographical coordinates. The next two lines are to coordinate the transformation from the system of coordinates to the system of projection of the active layer. Another piece of code starting with a loop performs the calculation of derivatives and distortion measurements, i.e. parametric scales, extreme scales and the Airy's measure. Then we use the three functions of matplotlib ie, figure (), contour () and show () to generate the isolines of distortion. The effect of this scheme is shown at figure 16.

Using a small modification to the code we can get a three-dimensional visualization of isolines fairly easily. Just enter two lines of the code:

$$
\begin{aligned}
& X=\text { fig.add_subplot }\left(111 \text { projection }=, 3 D^{\prime}\right) \\
& \text { ax.contour }(X, Y, Z)
\end{aligned}
$$

The effect has been shown at figure 17 . The coordinates of the cursor and the value of distortion are displayed at the bottom of the window.

Another way of visualization, that we can get with the help of this library, is a surface of distortions (fig. 18).

To achieve this effect, type the following two lines of the code:

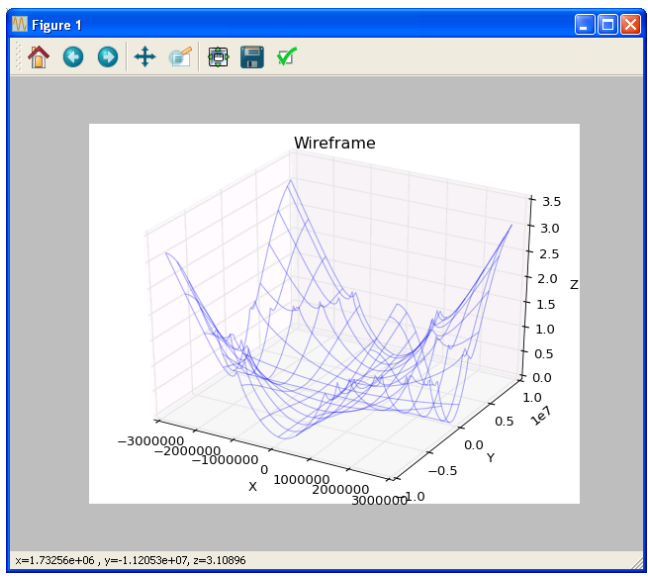

Fig. 19. The surface of distortions generated by developed plug-in

$$
\begin{aligned}
& x=\text { fig.add_subplot }(111 \text { projection }=\text { '3D') } \\
& \text { x.plot_surface }(X, Y, Z)
\end{aligned}
$$

Using wireframe () one can generate a similar effect, but in the form of a grid (fig. 19).

Just enter the following line of the code:

$$
\begin{aligned}
& \text { ax.plot_wireframe }(X, Y, Z \text {, rstride }=8 \text {, } \\
& \text { cstride }=8 \text { alpha }=0.3) .
\end{aligned}
$$

\section{Conclusions}

As you can see from this article, the distortions of cartographical projections can be presented in many different ways and the matplotlib library is a great tool, very helpful for this. The matplotlib library has many other possibilities for the visualization of calculation results, more, than it is shown in the article not only the cartographical distortions, but also the results of other analyzes. The main advantage is its simplicity and ease of visualization. A disadvantage is that the results are displayed in a separate window, and they are not stored as the layers. Such a solution would require the implementation of more advanced programming works. 


\section{Literature}

Balcerzak J., Panasiuk J., Pokrowska U., 1995, Wybrane zagadnienia z podstaw teorii odwzorowań kartograficznych. Warszawa: Oficyna Wydawnicza Politechniki Warszawskiej.

Biernacki F., 1949, Teoria odwzorowań powierzchni dla geodetów i kartografów. „Prace Geodezyjnego Instytutu Naukowo-Badawczego" Nr 4. Warszawa: Główny Urząd Pomiaru Kraju.

Bugejewski L., Snyder J., 1995, Map projections. A reference manual. Taylor \& Francis.

Chrisman, N., and J. F. Girres, 2015, First, do no harm: Eliminating systematic error in analytical results of GIS applications. In: "Uncertainty Modelling and Quality Control for Spatial Data”, pp. 27-41, CRC Press Taylor\&Francis Group.

Kimerling A., Overton W., White D., 1995, Statistical comparison of map projection distortion within irregular areas. "Cartography and Geographic Information Science” Vol. 22, no. 3, pp. 205-221.

Laskowski P., 1997, Distortion spectrum fundamentals. "Cartographica" Vol. 34, no. 3, pp. 3-15.

Mulcahy K.A., Clarke K.C., 2001, Symbolisation of map projection distortion: a review. "Cartography and Geography Information Science" Vol. 28, no. 3, pp. 167-181.

Pędzich P., 2005, Conformal projection with minimal distortions. In: „XXII International Cartographic Conference Proceedings", La Coruna.

Pędzich P., 2008, Wybrane aspekty konstruowania odwzorowań kartograficznych o możliwie najmniejszych zniekształceniach odwzorowawczych. „Roczniki Geomatyki” T. VI, T. 4, pp. 89-102.
Pędzich P., 2014, Podstawy odwzorowań kartograficznych z aplikacjami komputerowymi. Warszawa: Oficyna Wydawnicza Politechniki Warszawskiej.

Tobler W., 1986, Measuring the similarities of map projections. "American Cartographer" Vol. 13, no. 2, pp.135-139.

\section{Internet sources}

archive.org/details/mmoiresurlarepr00tissgoog

demonstrations.wolfram.com/DistortionsInMapProjections/

slvg.soe.ucsc.edu/map.html

projections.mgis.psu.edu/

matplotlib.org/users/screenshots.html\#mplot3d

sandbox.idre.ucla.edu/sandbox/intro-to-maps-historyand-beyond

www.colorado.edu/geography/gcraft/notes/mapproj/ mapproj_f.html

www.progonos.com/furuti/MapProj/Normal/TOC/ cartTOC.html

www.uni-stuttgart.de/gi/geoengine/mappro/

www.mathworks.com/help/map/visualizing-and-quantifying-projection-distortions.html

pl.wikipedia.org/wiki/Uk\%C5\%82ad_GUGIK_1980\#/ media/File:Gugik80_znieksztalcenia.png

www.progonos.com/furuti/MapProj/Dither/CartProp/ DefPatA/defPatA.html

www.oshermaps.org/of-maps-and-history/great-interactive-tool-to-teach-the-distortions-of-the-mercator-projection 